\title{
375. Die Chirurgie der benignen Struma
}

\author{
W. Wayand und W. Pimpl \\ I. Chirurgische Abteilung der Landeskrankenanstalten Salzburg (Vorstand: Prof. Dr. H. Steiner) \\ und Ludwig Boltzmann-Institut für Experimentelle und Gastroenterologische Chirurgie Salzburg-Hallein \\ (Leiter: Prof. Dr. H. Steiner, Prof. Dr. O. Boeckl), A-5020 Salzburg
}

\section{Surgery of Simple Goitre}

Summary. The historical development of thyroid surgery in Austria is closely associated with the names Billroth, Mikulicz, Breitner, Urban, Kaspar and Huber. Our surgical concept in a few words: The upper part of the patient's body raised at an angle of $60^{\circ}$; division of the thyroid isthmus; ligation and dissection of superior thyroid vessels; ligation of the inferior thyroid artery; preparation of the recurrent laryngeal nerve obligatory in recurrent goiter and malignoma; intracapsular resection of the gland; suction drainage of the operative field and suture clips, both for 3 days. Goitre prophylaxis is obligatory for the rest of the patient's life, depending on the preoperative morphology and function of the goitre. Results (complications, mortality) in 4,601 patients from 1964 to 1982.

Key words: Thyroid surgery - Historical development - Technique - Goitre prophylaxis.

Zusammenfassung. Die historische Entwicklung der Chirurgie der Struma ist für Österreich mit den Namen Billroth, Mikulicz, Breitner, Urban, Kaspar, Huber verbunden. Stichwortartig unser Operationskonzept: Oberkörper des Patienten um ca. $60^{\circ}$ aufgerichtet, querer Hautschnitt, Freilegung der Trachea durch Spaltung des Isthmus, Ligatur und Durchtrennung der oberen Polgefäße, Ligatur der A. tyreoidea inferior schilddrüsenfern, Darstellung des N. laryng. recurrens (bei Rezidivstrumen und Carcinomen obligat), intracapsuläre keilförmige Resektion, Redondrainage und Hautklammerung (beides für 3 Tage), obligate Rezidivprophylaxe, dabei individuelles Vorgehen je nach präoprativer Morphologie und Funktion der Struma. Darstellung der Ergebnisse an Hand von 4601 Patienten von 1964- 1982.

Schlüsselwörter: Benigne Struma - Historische Entwicklung - Operationskonzept - Rezidivprophylaxe.

\section{Beeinflußt die Pankreasgangocclusion mit Prolamin die Langerhans'schen Inseln beim Menschen?}

\author{
J. G. Doertenbach, H. J. C. Wenisch, H. Müller und A. Encke
}

Abteilung für Allgemein- und Abdominalchirurgie (Leiter: Prof. A. Encke) und Abteilung Pathologie II (Leiter: Prof. Stutte), Theodor-Stern-Kai 7, D-6000 Frankfurt 70

\section{Does Pancreatic Duct Occlusion by Prolamin Affect the Islets of Langerhans in Man?}

Summary. Aim of the clinical observation was to test whether, following pancreatic duct obstruction with Ethibloc, histomorphological alterations within the islets of Langerhans can be proved. - As in our experimental data, 6 months after sclerotherapy we can demonstrate distinct damage within the endocrine pancreas of a 31-year-old women. These findings can be shown histomorphologically, by immunohistochemical techniques and by electron microscopy. The beta-cell system appears endangered on a long-term basis. Different occlusion substances might be more suitable.

Key words: Islets of Langerhans - Pancreatic duct - Prolamin.

Zusammenfassung. Ziel dieser klinischen Beobachtung ist zu überprüfen, ob nach Pankreasgangocclusion mit Ethibloc unseren tierexperimentellen Befunden vergleichbare histomorphologische, immunohistochemische sowie ultrastrukturelle Veränderungen nachweisbar sind. Tatsächlich finden sich bei einer 31 Jahre alten Patientin 6 Monate nach Pankreasgangocclusion deutliche Zeichen cellulärer und ultrastruktureller Schädigungen innerhalb der Langerhans'schen Inseln. Dies deutet auf eine langfristige Gefährdung der Beta-Zellen und somit des Glucosestoffwechsels hin. Möglicherweise sind andere Occlusionssubstanzen geeigneter.

Schliusselwörter: Langerhans'sche Inseln - Pankreasgangocclusion - Prolamin. 